\title{
5. Zusammenfassung
}

Das Ende der massenhaften Flucht und Auswanderung aus der DDR ist bekannt: Als die Fluchtbewegung Anfang der sechziger Jahre wieder stark zunahm, ließen es die entscheidenden Kräfte auf der internationalen Bühne zu, daB die Führung der DDR WestBerlin einmauerte und damit das letzte Schlupfloch in die Bundesrepublik verschloB. Schwerbewaffnete Soldaten ersetzten die recht harmlosen Ost-Berliner Zöllner, die bislang eher „mit Steinchen FuBball“ gespielt, „sich als Narren“ aufgeführt und statt verdāchtiger Personen und Autos lieber jüngere[] weibliche[] Personen “ kontrolliert hatten, „obwohl hierzu überhaupt kein Anlaß vorhanden war ${ }^{4}{ }^{1}$ Wie im Westen der DDR gab es nun auch mitten in Berlin ein militarisiertes Grenzgebiet, das normale Bürger nur unter Todesgefahr betreten konnten.

Die Mauer unterband den Massenexodus, offenbarte aber gleichzeitig, daß die DDR der Konkurrenz aus dem Westen hoffnungslos unterlegen war. Gerade dieser rigorose Schritt im August 1961 weist auf die Grenzen der SED-Diktatur hin. Immerhin hatten seit 1945 etwa drei von weniger als 19 Millionen Einwohnern der SBZ/DDR ihrer staatlichen Obrigkeit den Gehorsam verweigert. Auch wenn die Flüchtlinge dank der deutschen Teilung ein Exil unter den denkbar günstigsten Bedingungen erwartete, blieb der illegale Weggang meistens mit einem Risiko und mit dem Verlust von Heimat, sozialen Bindungen und häufig auch von Eigentum verbunden. Daher zeigen Flucht und Abwanderung aus der DDR nicht nur, daß die nationale Einheit in den Köpfen der Menschen noch fortbestand; ebenso deutlich belegten sie die mangelnde Akzeptanz des Gesellschaftssystems der DDR durch die eigene Bevölkerung.

Opposition und Dissidenz wurden in den ersten Jahren der DDR selten offen artikuliert. Neben der zum Teil brutalen Unterdrūckung von tatsächlichen und vermeintlichen politischen Gegnern lag dies vorwiegend an der offenen Grenze. Hirschman hat darauf hingewiesen, daß Abwanderung gewöhnlich ein individueller Akt ist, der ohne öffentliche Artikulation vonstatten geht. Jede Republikflucht zeigte die Kommunikationsunfāhigkeit zwischen Regierenden und Regierten, zwischen Partei und Bevölkerung: Die meisten Menschen gingen heimlich, ohne ihre Gründe außerhalb des engsten privaten Kreises zu erlāutern. Neben der Situation in der DDR war die Attraktivităt des Westens die entscheidende Ursache der Flucht- und Abwanderungsbewegung. Selbst die SED-Führung konnte sich dieser deutsch-deutschen Konkurrenz nicht entziehen, so daß Ulbricht 1958 die $\mathbf{P a}$ role vom wirtschaftlichen ${ }_{n}$ einholen und überholen“ der Bundesrepublik innerhalb der nächsten drei Jahre ausgab. ${ }^{2}$ Entscheidend für das wirtschaftliche Hinterherhinken der DDR waren die radikalen gesellschaftlichen Veränderungen in den 16 Jahren zwischen

\footnotetext{
' Wiederholt kritisierten DDR-Berichte die zu oberflāchlichen Kontrollen in Berlin; Zitate aus: „Aktenvermerk über die Kontrolltätigkeit an der Sektorengrenze" vom 13.2. 1958 (BArch DO 1/11, Nr. 406, Bl. 68) und $Z$ wischenbericht der Überprüfung der Dienstdurchführung an der Sektorengrenze durch die Bereitschaften des PdVP Berlin" vom 22.4. 1959 (Dok. 11).

2 Weber, Die DDR (2006), S. 51; Staritz, Geschichte der DDR (1996), S. $173 \mathrm{ff}$.
} 
Kriegsende und Mauerbau. Dadurch blieben Regierung und SED die Zustimmung selbst von vielen sozialen Aufsteigern wie den Hochschulabgängern aus traditionell benachteiligten Schichten - versagt. Gegen diese Unzufriedenheit mit den individuellen Möglichkeiten in der DDR bot die Bundesrepublik vielversprechende Perspektiven, die sich hāufig mit der Hoffnung auf ein politisch und sozial ruhigeres Umfeld verbanden, in dem Arbeit und Privatleben ohne permanente politische Bezüge und öffentliche Bekenntnisse möglich sein würden.

Bis 1952 wurde die Abwanderung in den Westen in erster Linie als soziale Entlastung der Zusammenbruchsgesellschaft angesehen, durch die knappe Ressourcen wie Wohnung, Arbeit und Nahrung geschont wurden. In den ersten Nachkriegsjahren verließen viele Menschen die SBZ aufgrund unmittelbarer Kriegsfolgen, etwa wenn sie nach der Evakuierung in westliche Großstādte zurūckkehrten oder einen Neuanfang im außereuropäischen Ausland wagten. Darüber hinaus flüchteten viele Menschen aus der SBZ/DDR in den Westen, die von Besatzungsmacht, KPD/SED oder regierungsamtlichen Stellen vertrieben oder durch Repressionsmaßnahmen und -drohungen indirekt zum Weggang animiert wurden, weil sie als Mitglieder der alten Eliten angeblich dem Aufbau des Sozialismus im Wege standen. Vor allem die Bodenreform, die Enteignungen und die Entnazifizierung richteten sich rūcksichtslos etwa gegen Großgrundbesitzer, Industrielle und politische Gegner und zielten zum Teil direkt darauf, diese aus ihrer Heimat zu vertreiben. Eine ambivalentere Behandlung erfuhren viele Ärzte, Ingenieure und andere Mitglieder von Funktionseliten, auf deren Fachwissen und Arbeitskraft auch die neue politische Führung nicht verzichten konnte und wollte. Wie schon wāhrend der Entnazifizierung behandelte die SED diese Menschen mit einer gewissen Nachsicht und gewährte ihnen teilweise sogar schon früh wieder individuelle Privilegien, um sie in der SBZ/DDR zu halten - mit geringem Erfolg.

Trotz des geringen Interesses für die sozialen Verluste der massenhaften Flucht und Abwanderung beurteilte Ost-Berlin ihre politischen Implikationen schon in den ersten sieben Jahren nach Kriegsende als problematisch, weil jeder unerlaubte Weggang ein grundlegendes Defizit des ostdeutschen Staates und seiner Behörden augenfāllig machte: Ost-Berlin konnte weder die eigene Bevölkerung noch die eigenen Grenzen wirksam kontrollieren. Als die größten Wirren der Nachkriegszeit vorbei waren und die sowjetische Besatzungsmacht immer mehr Bereiche der Grenz-, Reise- und Meldepolitik an deutsche Behörden übergab, nahmen die Versuche zu, diese Bereiche staatlichen Handelns zu effektivieren. Neben dem allgemeinen Ziel, alle Bürger möglichst wirkungsvoll zu erfassen, zu kontrollieren und gegebenenfalls zu lenken, richtete sich der Ehrgeiz der SED seit der Staatsgründung vor allem darauf, die Eigenstaatlichkeit der DDR durch funktionierende Meldebehörden und die Kontrolle des eigenen Territoriums unter Beweis zu stellen. Die systematische Wahrnehmung der illegalen Abwanderung begann also, weil jede einzelne Flucht praktisch bestātigte, daß große Teile der internationalen Staatengemeinschaft ebenso wie der (ost)deutschen Bevölkerung die DDR nicht als eigenstāndigen Staat anerkannten.

Erst Mitte 1952 begann Ost-Berlin, neben dem politischen Prestigeverlust auch die gesellschaftliche Brisanz des Massenexodus wahrzunehmen. Ironischerweise führten erst der Ausbau der Grenzanlagen im Westen der DDR und die damit verbundene Zwangsaussiedlung zahlreicher Menschen aus der $5 \mathrm{~km}$-Sperrzone zu dieser größeren Aufmerksamkeit. Mit der Verschärfung und Militarisierung des Grenzregimes hatten SED und Besatzungs- 
macht zwar weniger auf den illegalen Grenzverkehr als auf die internationale Politik reagiert: Wie es bald darauf auf der Zweiten Parteikonferenz der SED hieB, ging es darum, den "Aufbau des Sozialismus" voranzutreiben und damit die Eigenstaatlichkeit der DDR zu untermauern, wozu auch die seit Kriegsende von der SEDSpitze mit MiBtrauen beāugten West-Ost-Kontakte weitgehend eingeschränkt werden sollten. Die erste Folge der verstārkten Überwachung der Grenze war aber, daß viele der Zwangsausgesiedelten nicht nur das Sperrgebiet verließen, sondern gleich die DDR. Schon bald forderte die Besatzungsmacht von den deutschen Behörden Rechenschaft über diese Fluchtwelle, wobei es auch der SKK noch in erster Linie um Prestigeverlust im Westen ging.

Da das verschärfte Grenzregime nicht den Hōhepunkt, sondern nur einen Teil der politischen Radikalisierungen von 1952/53 darstellte, stieg bald darauf die Zahl der Flüchtlinge insgesamt stark an. Die auf der Zweiten Parteikonferenz beschlossene "planmäBige Errichtung der Grundlagen des Sozialismus" führte mit ihren gezielten Maßnahmen gegen Selbstāndige und politische Gegner zu einem dramatischen Anstieg der Fluchtbewegung. Die 1952 in den ersten Analysen von Partei- und Regierungsbehörden über die Republikflucht noch gehegte Hoffnung, die deutsch-deutsche Flucht- und Wanderungsbewegung zugunsten der DDR lenken zu können - Leistungsschwache und politische Gegner also an den Westen abzugeben und hochqualifizierte Fachleute von dort anzuwerben -, ließ sich ganz offensichtlich nicht realisieren. Im Laufe der Jahre wurde eher das Gegenteil offenbar: Die DDR verlor in erster Linie junge und hochqualifizierte Arbeitskräfte, während die Zuwanderer aus dem Westen von den verantwortlichen Behörden immer stärker als soziale Belastung und kriminelle Gefahr angesehen wurden.

Die Republikflucht war zwar nur ein stummer Ausdruck der gesellschaftlichen Unzufriedenheit, aber er wurde besonders gut verstanden: So bezog sich die Besatzungsmacht Anfang Juni 1953 in ihren Anweisungen an die SED, die Umgestaltungspolitik zu mäßigen und einen Teil der unpopulären MaBnahmen zurückzunehmen, ausdrücklich auf Republikflucht. Ost-Berlin kam der Weisung nach und beschloß - kurz vor den Unruhen Mitte Juni 1953 - ihren „Neuen Kurs“: Zahlreiche wirtschaftspolitische Maßnahmen wurden zurückgenommen, der politische Kampf gegen Andersdenkende gemäßigt und Republikflüchtige mit diversen Angeboten und Versprechungen zur Rūckkehr aufgefordert. Die Liberalisierung bedeutete vor allem für solche Bürger der DDR eine Entspannung, die unmittelbar im Visier der SED-Politik gestanden hatten. Selbständige, Christen und andere potentielle Zielscheiben rigoroser SED-Maßnahmen, aber auch viele andere Bürger stellten daraufhin ihre Pläne zur Abwanderung in den Westen zurück, so daß der Neue Kurs" einen unmittelbaren Erfolg verzeichnen konnte, nämlich den drastischen Rückgang der monatlichen Fluchtzahlen.

Die SED hielt den liberaleren Kurs aber nicht lange durch. Nachdem die Mischung aus militärischer Unruhebekämpfung und gezielten Zugeständnissen vom Sommer 1953 ihre Machtposition bald wieder gesichert hatte, zeigte sich der bloB taktische Charakter der Liberalisierung. Schritt für Schritt kehrten nicht zuletzt viele regionale und lokale Funktionstrāger zur alten Politik zurück, ohne daß dazu offizielle Entscheidungen wie auf der Zweiten Parteikonferenz gefällt wurden. Die Reaktion der Bevölkerung blieb auch dieses Mal nicht aus: Spātestens 1955 war der erneute Anstieg der Fluchtzahlen nicht mehr zu übersehen und zunehmend mehr Institutionen ãußerten ihre Besorgnis über den Verlust wichtiger Fachkräfte, deren plötzlicher Weggang vor allem in die Bereiche Wirtschaft, Wissenschaft und Gesundheitsversorgung große Lücken riß. 
Die hauptsāchlich mit der Beobachtung und Bekāmpfung von Flucht und Abwanderung beschäftigten Behörden der Volkspolizei, der Innenverwaltung und der Abteilung Staat und Recht beim ZK der SED versuchten, dem Problem durch verschiedene Maßnahmen Herr zu werden, die sich schon 1952/53 als nicht erfolgreich erwiesen hatten und auch Mitte der fünfziger Jahre nur wenige Fluchtwillige vom Weggang in den Westen abhielten. Dazu zāhlten vor allem die Versuche, Westdeutsche zum Umzug in die DDR und bereits Geflohene zur Rūckkehr zu bewegen. Obwohl die Statistiken etwa 600000 Zuziehende und Rūckkehrer aus dem Westen zählten, ${ }^{3}$ hielt sich der Erfolg quantitativ wie qualitativ in engen Grenzen. Abgesehen von der Anzahl und dem niedrigen Qualifikationsniveau der Zuwanderer, durch das die Republikflüchtigen nicht ersetzt werden konnten, lag der Mißerfolg an der schlechten Behandlung der Zuwanderer: Sie begann mit dem wiederholt beklagten unfreundlichen Empfang an der Grenze und setzte sich zum Teil jahrelang fort, da die ehemals Westdeutschen politisch verdāchtigt und polizeilich überwacht wurden. Vor allem diejenigen, die in den ersten Jahren aufgrund der Arbeitslosigkeit in der Bundesrepublik in die DDR kamen, gingen hãufig in den Westen zurück, da sie dort beruflich und privat weniger Einschränkungen unterlagen. Ein Teil ihres Unmuts entstand auch durch die dauerhafte Werbung und Propaganda gegen den Westen, durch die die Bürger der DDR davon überzeugt werden sollten, im besseren Teil Deutschlands zu leben, der dem ,reaktionären' Westen nicht nur politisch-moralisch, sondern langfristig auch wirtschaftlich überlegen sei. Das Zentrum der Propaganda gegen die Westflucht war die Behauptung, daB die DDR ihren Bürgern die besseren Perspektiven bieten könne. Ein großer Teil der Menschen vertraute jedoch der eigenen Anschauung mehr als dieser Propaganda und konnte das Ausmaß des westdeutschen Wirtschaftsbooms, der dem größten Teil der dortigen Bevölkerung zugute kam, nicht übersehen. Um solchem Unmut entgegenzuwirken, bemühten sich amtliche Stellen zum einen um weitere Einschrānkungen von Westkontakten, zum anderen nahmen sie die Propaganda zum Lob des eigenen Staates etwas zurück. Statt dessen verpflichtete die SED-Führung die Medien zur regelmãßigen Berichterstattung darūber, daß die ehemaligen Bürger der DDR im Westen in Arbeitslosigkeit und Elend leben mußten. Hinweise darauf, daß diesen Geschichten mehr Glauben geschenkt wurde, lassen sich nicht finden.

Neben diesen propagandistischen Versuchen, der Fluchtbewegung Herr zu werden, bemūhten sich die Behörden der DDR auch um praktische Maßnahmen zur Verhinderung von Republikfluchten. Diese waren häufig auf einzelne Personen oder Berufsgruppen bezogen. So erhielten viele Fachleute und Spezialisten Sondervergünstigungen materieller oder finanzieller Art wie Autos, bessere Wohnungen und höhere Gehālter; nicht selten wurden West- und Urlaubsreisen vereinfacht und unterstützt, einzelne Sanktionen und Beschrānkungen wie das Studierverbot für Akademikerkinder zurūckgenommen oder wissenschaftliche Zusatztitel vergeben. Die Wirkung dieser Maßnahmen war in den fünfziger Jahren ebenso schwer abzuschātzen wie heute, denn zahlreiche Fachleute verließen trotz solch konkreter Zugestāndnisse die DDR, zumal der bundesrepublikanische Arbeitsmarkt seit Mitte der fünfziger Jahre dem größten Teil der berufstātigen Bevölkerung Arbeits- und Lebensbedingungen bot, die die angebotenen Privilegien weit überragten. Parallel zu solchen Angeboten und Vergünstigungen reagierten die Behörden auch stets mit einer Anzahl von restriktiven und repressiven Methoden auf die Fluchtbewegung. In

\footnotetext{
s Zahl für die Jahre 1949 bis 1961, nach Schmelz, West-Ost-Migration (2002), S. 19.
} 
erster Linie gehōrten dazu die Beobachtung, Überwachung und Kontrolle von verdāchtigen Personen. Zu diesem Zweck wurde ein umfangreiches Melde- und Karteiwesen eingerichtet, in dem verdāchtige Personengruppen wie Grenzgānger, zurückgekehrte Republikflūchtlinge, hãufige Antragsteller für Westreisen oder Angehörige von Republikflüchtlingen erfaßt werden soliten. Darüber hinaus registrierten die Meldebehörden noch zahlreiche weitere Aktivitãten, die auf Fluchtabsichten hinweisen konnten. Das wichtigste Ergebnis dieser Bemūhungen waren dauerhafte Klagen darüber, daB die erhobenen Informationen nicht operationalisierbar waren, zumal einzelne dieser MaBnahmen die vermeintlich Fluchtwilligen oft erst in den Westen trieben. Ganz offensichtlich konnten die in erster Linie für die Bekämpfung der Abwanderung verantwortlichen Abteilungen für Innere Angelegenheiten und die Polizei das Problem nicht beseitigen. Sie wandten die entsprechenden Maßnahmen gegen die Fluchtbewegung zwar bis zum Mauerbau an, verfaßten im Laufe der fünfziger Jahre aber immer häufiger programmatische Berichte und Appelle gegen den "Ressortgeist ${ }^{“}$ einzelner Behörden, Betriebe und anderer Institutionen der DDR, die durch ihr Verhalten ebenfalls zur Fluchtbewegung beitrugen, ihrer Bekämpfung aber zu wenig Aufmerksamkeit widmeten. Die Versuche, die Ost-West-Flucht etwa durch die Errichtung von ressortübergreifenden Kommissionen zu einem politischen Querschnittsthema zu machen, scheiterten durchweg.

Ende 1957 nahm Ost-Berlin erneut eine deutliche Kursānderung in der Grenz- und Reisepolitik vor. Anders als Mitte 1953 beschlossen Partei und Regierung allerdings keine Liberalisierung, sondern zwei entscheidende Einschränkungen der Reisemöglichkeiten: Den bis dahin nur indirekt bestrafbaren unerlaubten Grenzübertritt in die Bundesrepublik erklärte die Volkskammer durch das Paßãnderungsgesetz vom 11. Dezember 1957 zum eigenen Straftatbestand, und etwa seit dem gleichen Zeitpunkt genehmigte die Polizei nur noch einen Bruchteil der zuvor bewilligten Besuchsreisen in den Westen. Die Kursänderung wurde durch politisierte Strafverfahren ergänzt, in denen die Gerichte selbst belanglose Grenz- und Reisevergehen mit zum Teil hohen Haftstrafen ahndeten. Die erwartete abschreckende Wirkung ließ nicht lange auf sich warten: Ebenso wie nach den Liberalisierungen 1953 gingen die Fluchtzahlen auch infolge des repressiven Maßnahmenkatalogs deutlich zurūck. Doch der Erfolg war nicht von Dauer. Ebenso wie nach dem Einschnitt vom Sommer 1953 stieg die Zahl der Westflüchtlinge nach dem statistischen Rekordtief von 1959 seit Anfang 1960 wieder an. Durch das Verbot von legalen Besuchsreisen in den Westen, von denen viele DDR-Bürger nicht zurückgekehrt waren, nahm der illegale Weggang über die Grenze in Berlin zu, über deren Schließung dadurch im Zuge der zweiten Berlinkrise vermehrt Gerüchte im Umlauf waren. Trotz der hãufig zitierten offiziellen Versicherung Ulbrichts, daß niemand vorhabe, eine Mauer zu bauen, ${ }^{4}$ entschied sich Ost-Berlin für genau dieses Mittel, um das Ausbluten der eigenen Gesellschaft zu verhindern.

Seitdem die Behörden der DDR die kritischen Auswirkungen der Republikflucht in einzelnen gesellschaftlichen Bereichen erkannt hatten, erstellten die HVDVP, die Hauptabteilung Paß- und Meldewesen, die Abteilung Staat und Recht sowie zahlreiche weitere Institutionen viele Berichte über den Umfang und die Ursachen der Abwanderung. Dazu wurden Informationsbrigaden in einzelne Kreise, Behörden oder Betriebe geschickt, die sich mit den Verhāltnissen vor Ort und den Fluchtgründen der jeweiligen Mitarbeiter ver-

4 So auf einer Pressekonferenz am 15. 6. 1961, ND Nr. 164 v. 16. 6. 1961, S. 4. 
traut machen und teilweise auch die Funktionäre vor Ort im Kampf gegen die Fluchtbewegung schulen sollten. Bereits 1952 erhielt das Sekretariat des ZK entsprechende Berichte, die das breite Tableau der Fluchtursachen auflisteten und mit Einzelbeispielen illustrierten. Solche Berichte häuften sich im Laufe der folgenden Jahre: In immer neuen Varianten hielten sie die immer gleichen Vorfalle und Ursachen fest, so daß ein Brigademitglied Mitte 1960 resigniert darüber klagte, ${ }_{n}$ da $B$ immer wieder dieselben Māngel festgestellt worden sind."s

Trotz ihrer Redundanz lieferten die Berichte zahlreiche Hinweise auf Probleme der DDR-Gesellschaft, die bei den Bürgern zu Unzufriedenheit führten und viele zur Flucht veranlaBten. Die Analysen legten ihr Augenmerk hauptsächlich auf berufsspezifische und allgemeine Fluchtgründe, wobei die Darstellung von Einzelfallen und die Vermittlung von Gesamteindrücken durchweg wichtiger war als eine strenge Orientierung an prāzisen gesellschaftswissenschaftlichen Kategorien. Dadurch gerieten beispielsweise nicht berufstātige Ehefrauen in die Berufskategorie ihrer Ehemänner, Facharbeiter und andere nicht akademisch ausgebildete Spezialisten konnten in Berichten über einzelne Betriebe als "Intelligenzler" behandelt werden, und bei den Bauern und Handwerkern wurde in einem Fall zwischen den Selbstāndigen und den Genossenschaftsmitgliedern unterschieden, im anderen Fall nicht.

Die Konzentration auf einzelne Berufsgruppen unterstreicht, wie stark die SED die Būrger der DDR an ihrer Produktivitāt und damit an ihrer Leistungsfăhigkeit für den Aufbau des Sozialismus maß, was sich auch am überdurchschnittlich hohen Anteil an genehmigten Verzügen von alten und behinderten Menschen in den Westen zeigte. Einen ähnlichen Eindruck hinterlāßt die Intensitāt der Aufmerksamkeit für die verschiedenen Berufsgruppen: Obwohl sich die DDR als Arbeiter- und Bauernstaat verstand und sich die Korrektur der historisch bedingten Benachteiligungen für die Mitglieder gerade dieser Berufsgruppen auf die Fahnen geschrieben hatte, wurden ihre Fluchtmotive und -umstānde weit weniger detailliert beschrieben als die der Fachleute und Akademiker. Dies hatte vor allem zwei Gründe: Zum einen waren die Verluste und Probleme größer und einfacher zu erkennen, wenn Akademiker oder andere Spezialisten ihre Betriebe, Arztpraxen oder Schulen verließen, da es an Ersatzpersonal mangelte. Zum anderen hinterließen die Angehörigen dieser Berufsgruppen mit entsprechend höherer Schulbildung eher Stellungnahmen zu ihrer Flucht, die von den Berichterstattern zitiert und ausgewertet werden konnten, als Bauern und Arbeiter.

Inhaltlich spiegeln alle Berichte die Unzufriedenheit in der DDR über die Arbeitsbedingungen und den Lebensstandard. Wirtschaftlich Selbstāndige klagten über Benachteiligungen gegenūber den hãufig weit weniger produktiven Genossenschaften, sie berichteten von politisch motivierten Schikanen und der mangelhaften Belieferung mit Rohstoffen, Maschinen und anderen Gütern, ohne die sie ihre Arbeiten nicht verrichten konnten. Meistens verdankten sich die Mißstände einer Mischung aus politischem Rigorismus und fachlichem Unvermögen, etwa wenn den Bauern Saatgut oder Düngemittel erst kurz nach dem Zeitpunkt geliefert wurde, an dem dies landwirtschaftlich geboten gewesen wãre. Auch in den Darstellungen über einzelne Industriebetriebe, Krankenhäuser, Forschungslabors oder sonstige staatseigene und genossenschaftliche Betriebe führte die Unfăhigkeit von Fūhrungskadern hãufig zum Weggang von Mitarbeitern aller Ausbildungs-

${ }^{5}$ SPK, Chemiekaderkommission. am 28. 7. 1960 (BArch DO 1/34, Nr. 21725). 
stufen. Führungspersonal und Wissenschaftler ärgerten sich hãufig über fachfremde Tãtigkeiten infolge der Politisierung der Betriebe, durch die sie von ihren eigentlichen Aufgaben abgehalten wurden. Arbeiter beklagten etwa Materialmangel und Managementfehler, wodurch lange Wartezeiten entstanden, in denen sie hāufig unterhalb ihrer Qualifikation eingesetzt wurden.

Maßstab für Kritik und Unzufriedenheit war immer der Westen, in dem andere und letztlich effizientere Zustände vermutet wurden; nicht selten wurde auch auf die Arbeitsbedingungen in den Betrieben vor 1945 Bezug genommen, die inzwischen zum Teil im Westen neugegründet worden waren und die nach ihren ehemaligen, in der DDR verbliebenen Mitarbeitern Ausschau hielten. Der Blick in den Westen prägte auch die Darstellung der Fluchtgründe, die nicht unmittelbar mit der Arbeitswelt zu tun hatten. Viele Berichte formulierten sie vorwiegend im Zusammenhang mit der Unzufriedenheit und den Fluchtabsichten von Akademikern, die Probleme spürten aber - abgesehen von spezifischen Benachteiligungen wie dem Studienverbot für Akademikerkinder - fast alle Bevōlkerungsgruppen. Egal ob es um die Höhe von Gehältern und Löhnen, Wohnungsprobleme, Versorgungsengpässe oder um Reisemöglichkeiten ging, immer wieder spiegelte sich das Gefühl vieler Menschen in der DDR, im Vergleich zu ihren Familienangehörigen, Bekannten und ehemaligen Kollegen im Westen unter schlechteren Bedingungen zu leben.

Der verbreitete Eindruck der strukturellen Benachteiligung verstärkte sich noch durch den Anspruch der SED und ihrer Funktionäre, Wertungen und Weisungen für alle Lebensbereiche aussprechen zu kōnnen. In erster Linie spürte die Bevölkerung das bei Fragen des politischen und religiösen Bekenntnisses und bei Reiseanträgen, da die Vertreter der staatlichen Institutionen hier hăufig reflexhaft reagierten, ohne zwischen privatem Familienleben, wirtschaftlicher Produktivitât und politischer Loyalitāt zu unterscheiden. Trotz ihrer Detailfreude vermochten es die meisten Berichte daher nicht, die Probleme, die AnlaB zur Flucht gaben, systematisch zu ordnen und handlungsorientiert zu gewichten. Statt dessen reproduzierten sie immer wieder die dichotomen Muster von Klassenkampf und Systemgegensatz, wodurch sogar private Angelegenheiten wie Erbschaften, die Teilnahme an Beerdigungen oder die Reise zu Verwandten im Westen als politische Bekenntnisse überbewertet wurden. Selbst gewōhnliche Generations- und Schulkonflikte konnten im Dickicht der politischen Rhetorik zu unlōsbaren Problemen werden, wenn Jugendliche beispielsweise Schlager von Radio Luxemburg sangen oder Gefallen an westlichen "Schundromanen" oder Kinofilmen äußerten.

Die Erklärungen für die Fluchtbewegung, die SED und Staatsfunktionäre immer wieder anboten und die über die politische Beschimpfung der Abgewanderten hinausgingen, zeigen die Unfahigkeit im Umgang mit eigenen Fehlern und innenpolitischen Problemen. Wurden einzelne Kritikpunkte wie die als unzureichend empfundene Versorgung mit Gütern des täglichen Bedarfs nicht banalisiert, dann lieferten die Berichterstatter gewöhnlich zwei Erklārungsmuster, mit denen die Verantwortung von der SED, ihrem Staat und dessen Strukturen weggeschoben wurde. Entweder individualisierten sie die Verantwortung, indem sie einzelne Verwaltungsmitarbeiter und ihre angeblich zu bürokratische Arbeitsweise für Fehlentwicklungen, Überspitzungen und Unzufriedenheiten verantwortlich erklärten, oder sie externalisierten die Verantwortung unter den Stichwort der „Abwerbung “. Dabei ignorierten sie die historischen Wurzeln des trotz der politischen Teilung fortbestehenden gesamtdeutschen Arbeitsmarktes und erklārten jedes Angebot aus dem Westen, jeden Berufswechsel zu einer bundesrepublikanischen Firma oder Behörde zum 
Nachweis des aggressiven westlichen „Menschenhandels“. In ihrer ideologisch bedingten Unfāhigkeit, die Funktionstūchtigkeit von gesellschaftlichen Teilbereichen jenseits der unmittelbar politischen Sphäre wahrzunehmen, verstanden sie jede der vorwiegend betriebswirtschaftlich motivierten „Abwerbungen“ und Anstellungen als zentral geleitete Initiative Bonns zur Schādigung der Wirtschaft der DDR. Beide Erklārungsansātze trafen zwar einen Teil der gesellschaftiichen Wirklichkeit, verhinderten aber einen anatytischen Blick auf die Situation in der DDR und verschärften damit das antagonistische Klima, aufgrund dessen so viele Menschen die DDR verließen.

Da die Flucht- und Abwanderungsbewegung im allgemeinen, jeder Einzelfall aber auch im besonderen, letztlich nur in den antagonistischen Kategorien des internationalen Systemgegensatzes wahrgenommen wurden, konnten die Berichte keine Kategorien liefern, mit denen konkrete Politik und wirkungsvolle Schritte gegen die Flucht hätten eingeleitet werden kōnnen. Unklar ist, ob ein offenerer Umgang mit den Problemen und der Abschied von diesem dichotomen Weltbild die Herrschaft der SED untergraben hãtte. Immerhin schätzte die Mehrheit der Bevölkerung den westlichen Konsum und die nationale Einheit mehr als die Herrschaft der SED und die Eigenstaatlichkeit der DDR, denn diese Orientierung zeigte sich selbst bei der Jungen Intelligenz" und anderen Menschen, die von der sozialen Aufstiegsmobilităt in der DDR profitiert hatten und dennoch in den Westen gingen. Diese Unfāhigkeit der SED, Probleme und Konflikte im begrenzten Rahmen zu thematisieren, ohne auf ihre totalisierenden Gesellschaftskonzepte zurūckzugreifen, verstärkte aber noch die Tendenz zur Republikflucht, da dadurch aus punktuellen Unzufriedenheiten hăufig strukturelle wurden, für die es keine Lōsungsperspektiven mehr gab: „Wer nun ging, brauchte dazu kaum einen einzelnen tagespolitischen AnlaB, und nicht einmal alle zusammen: er war mit der DDR fertig, er glaubte ihr kein Wort mehr, er konnte nicht mehr mit ihr, er mochte ihre Stimme nicht mehr ertragen, ihm war die Luft aus dem Vertrauen gelassen worden, es ging nicht mehr". ${ }^{6}$

Die Westflucht ermöglichte eine konfliktfreie Beendigung von Problemen, die die individuelle und kollektive Einübung der Bewältigung von gesellschaftlichen Konflikten verhinderte. Dies verstārkte nicht nur die politische Zurückhaltung der Bevölkerung, die seit 1933 bereits unter der zweiten Diktatur lebte, sondern auch die besondere Halsstarrigkeit der SED. So hielt sich innerhalb der SED und der staatlichen Behörden bis Ende der fünfziger Jahre die Überzeugung, daß Flucht und Abwanderung neben dem Verlust an Arbeitskräften und Fachwissen auch eine Form des sanften Bürgerkriegs seien: Hatten Besatzungsmacht und KPD 1945 politische Gegner vertrieben, so qualifizierten sich die Republikflūchtigen in den folgenden Jahren durch ihren Schritt selbst als solche. Wie stark ideologisiert diese Wahrnehmung - ebenso wie die gesamtdeutsche Rhetorik - war, zeigt sich daran, daß sich niemand in den (hefetagen in Ost-Berlin darüber Gedanken machte, wo die in den Westen vertriebenen und geflüchteten Klassengegner bleiben sollten, wenn die immer wieder gescholtene Teilung aufgehoben sein würde. Auch wenn sie nicht direkt ausgesprochen wurde, erkannte die SED immerhin die Ambivalenz der Auswanderung. die ihr neben den Verlusten auch eine politische Entlastung und darüber hinaus vielen Bürgern der DDR Möglichkeiten des sozialen Aufstiegs bot, die sonst undenkbar gewesen wären. Indirekt stabilisierten die Republikflüchtigen damit nicht nur die politische, sondern auch die soziale Lage in der DDR.

\footnotetext{
6Johnson, Versuch (1970). S. 125.
} 
Trotz der fast durchweg erfolglosen Versuche der SED und ihres Staates, die Abwanderung in den Westen entscheidend einzuschränken, waren die Folgen der vorwiegend restriktiven und repressiven Grenz- und Reisepolitik nicht zu übersehen. Innerhalb weniger Jahre setzte die SED die Prioritāt des internationalen Systemgegensatzes vor dem nationalen Zusammenhalt durch. Die systematische Behinderung von Kontakten zwischen den beiden deutschen Staaten und die entsprechende politische Propaganda wirkten sich besonders schnell bei den Mitarbeitern der Grenz- und Sicherheitsongane aus, aber auch bei vielen Mitgliedern der SED, die dadurch nicht nur nationale, sondern auch familiäre Beziehungen gegenūber der politischen Frontstellung zurūckstellten. Mit ihrer schrittweisen Verschärfung der Reise- und Grenzpolitik bis zum Mauerbau forcierte die DDR damit den Paradigmenwechsel in der gesamtdeutschen Nachkriegsgeschichte: Während sich in der Bundesrepublik die Westbindung dank der materiellen und politischen Erfolge zunehmend stärker vor die gesamtdeutschen Fragen schob und die nationale Identität auflockerte, so forcierte die SED die gleiche Entwicklung damit, daB sie gewachsene menschliche Beziehungen mit Hilfe von politischen Verdächtigungen, wachsenden Kontakt- und Reiseverboten und schließlich mit dem Bau der Mauer zerstörte. 


\section{0}

